\title{
Editorial
}

\section{Catalyst Nanomaterials}

\author{
Ping Xu, ${ }^{1}$ Bo Song, ${ }^{2}$ Hongmei Luo, ${ }^{3}$ Ling Fei, ${ }^{4}$ and Hsing-Lin Wang ${ }^{5}$ \\ ${ }^{1}$ Department of Chemistry, Harbin Institute of Technology, Harbin 150001, China \\ ${ }^{2}$ Academy of Fundamental and Interdisciplinary Sciences, Harbin Institute of Technology, Harbin 150001, China \\ ${ }^{3}$ Department of Chemical and Materials Engineering, New Mexico State University, Las Cruces, NM 88003, USA \\ ${ }^{4}$ School of Chemical and Biomolecular Engineering, Cornell University, Ithaca, NY 14853, USA \\ ${ }^{5}$ C-PCS, Los Alamos National Laboratory, Los Alamos, NM 87544, USA
}

Correspondence should be addressed to Ping Xu; pxu@hit.edu.cn

Received 9 July 2015; Accepted 9 July 2015

Copyright (C) 2015 Ping Xu et al. This is an open access article distributed under the Creative Commons Attribution License, which permits unrestricted use, distribution, and reproduction in any medium, provided the original work is properly cited.

Catalyst nanomaterials are essential to life sciences, chemical engineering processes, environmental protection, and energy and fuels. Researches on catalyst materials include new approaches to synthesizing conventional catalysts, discovery of new catalysts, novel mechanistic studies on catalysis, and practical enhancements of known chemical or biological processes. In this special issue the published works are briefly addressed as follows.

The paper from South China University of Technology, China, reports the synthesis of a platinum monolayer coreshell catalyst with a ternary alloy nanoparticle core of Pd, Ir, and Ni. A Pt monolayer is deposited on carbon-supported PdIrNi nanoparticles using an underpotential deposition method. The core-shell Pd1Ir1Ni2@Pt/C catalyst exhibits excellent oxygen reduction reaction activity, with a mass activity significantly higher than that of Pt monolayer catalysts containing PdIr or PdNi nanoparticles as cores and four times higher than that of a commercial $\mathrm{Pt} / \mathrm{C}$ electrocatalyst.

The paper from Joint Institute for High Temperatures of Russian Academy of Sciences, Russia, studies the direct synthesis of porous multilayered graphene materials using thermal plasma at low pressure. Specific surface area and pore system of the synthesized graphene are comparable with properties of materials used as carriers of catalytically active components, such as alumina, silica-alumina, and activated charcoal.

The paper from The University of Kitakyushu, Japan, is about the synthesis of hydrocarbons from $\mathrm{H}_{2}$-deficient syngas in Fischer-Tropsch synthesis with coupled Co-based and Fe-based catalysts. Among the catalysts, the hybrid catalyst containing the Mn-added Fe-based catalyst exhibited the highest activity for the $\mathrm{CO}$ hydrogenation and the water-gas shift (WGS) reaction with the CO conversion of $45.0 \%$, the space time yields (STY) of hydrocarbons of $2.61 \mathrm{~mol} \mathrm{~kg}^{-1} \mathrm{~h}^{-1}$, and the STY of $\mathrm{CO}_{2}$ of $1.92 \mathrm{~mol} \mathrm{~kg}^{-1} \mathrm{~h}^{-1}$ after $6.5 \mathrm{~h}$ of the reaction.

The paper from Harbin Institute of Technology, China, focuses on the fabrication of $\mathrm{Fe}^{3+}$-exchanged titanate nanotubes (Fe-TNTs), which act as a new kind of highly active heterogeneous catalyst for Friedel-Crafts type benzylation. Fe-TNTs showed excellent catalytic activities in the benzylation of benzene and benzene derivatives. The recycling tests for Fe-TNTs were also carried out, where the reason for the gradually decreased activity was carefully investigated. The catalytic ability of used Fe-TNTs could be easily recovered by ion exchange again, indicating that Fe-TNTs were a highly active and durable heterogeneous catalyst for Friedel-Crafts type benzylation.

The paper from University of Malaya, Malaysia, talks about the preparation of mesoporous silica-supported chiral amino alcohols for the enantioselective addition of diethylzinc to aldehyde and asymmetric transfer hydrogenation to ketones. The developed catalytic process yielded optically active secondary aromatic alcohols with 92-99\% conversion and $70-82 \%$ enantioselectivity.

The paper from University Politehnica of Bucharest, Romania, is about influence of silver nanoparticles (AgNPs) on the photocatalytic activity of hybrid materials based on $\mathrm{TiO}_{2} \mathrm{P} 25$. 
The film manufactured from a physical mixture of $\mathrm{TiO}_{2} \mathrm{P} 25$ and AgNPs dispersion showed that the size distribution of the AgNPs was proved to be a key factor in determining the photodegradation activity of methyl orange.

\author{
Ping Xu \\ Bo Song \\ Hongmei Luo \\ Ling Fei \\ Hsing-Lin Wang
}



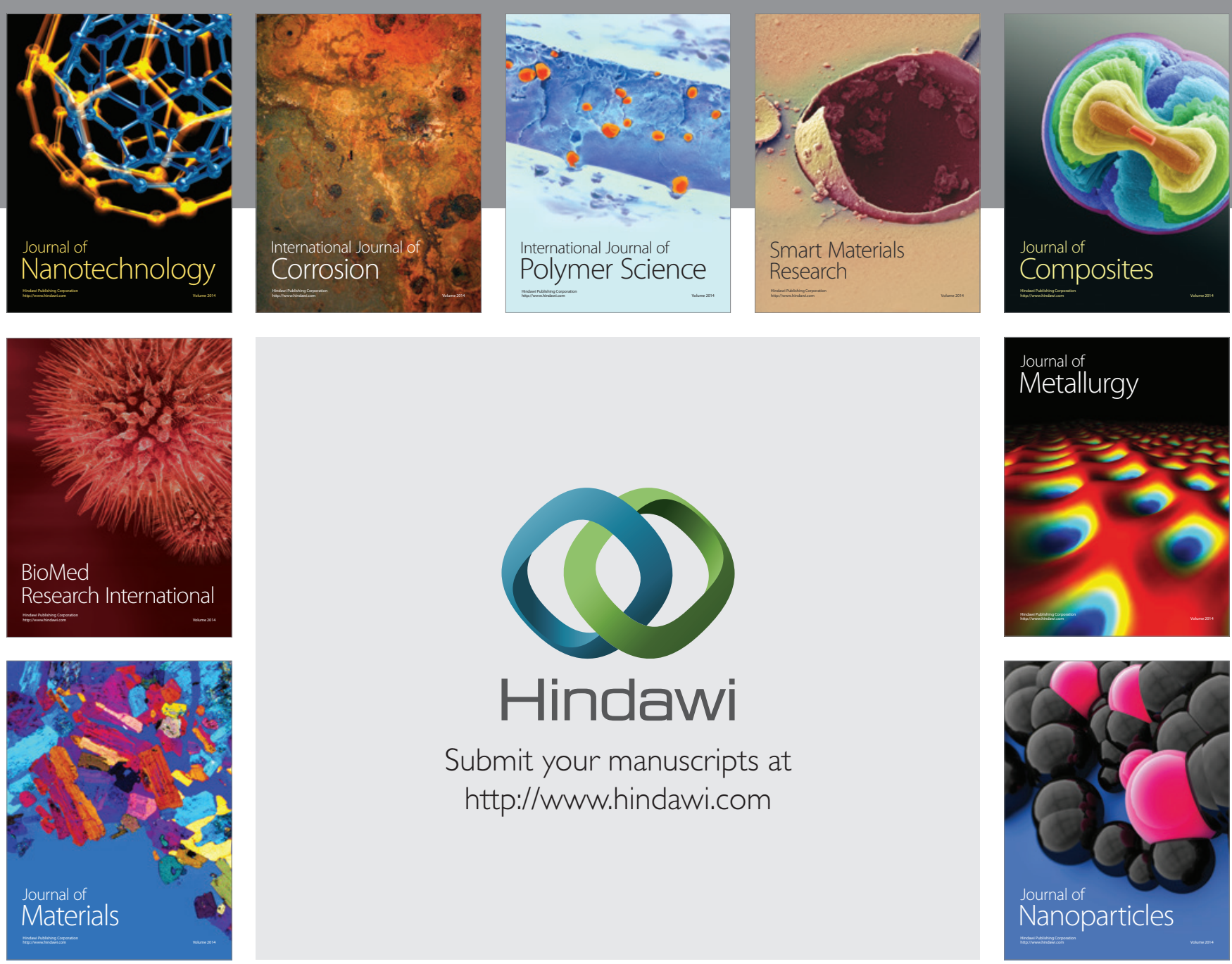

Submit your manuscripts at http://www.hindawi.com
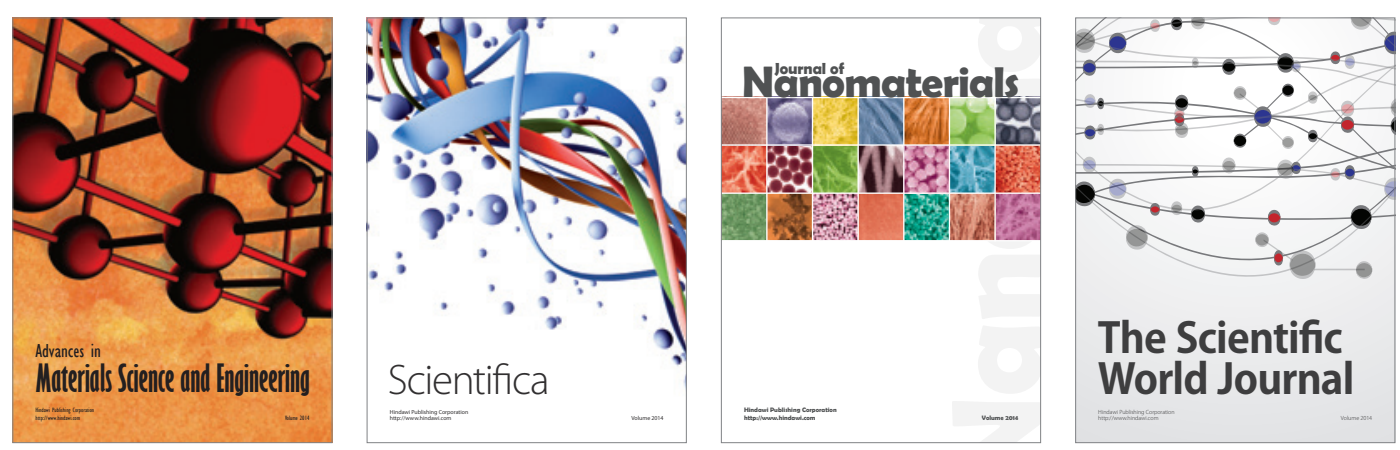

\section{The Scientific World Journal}
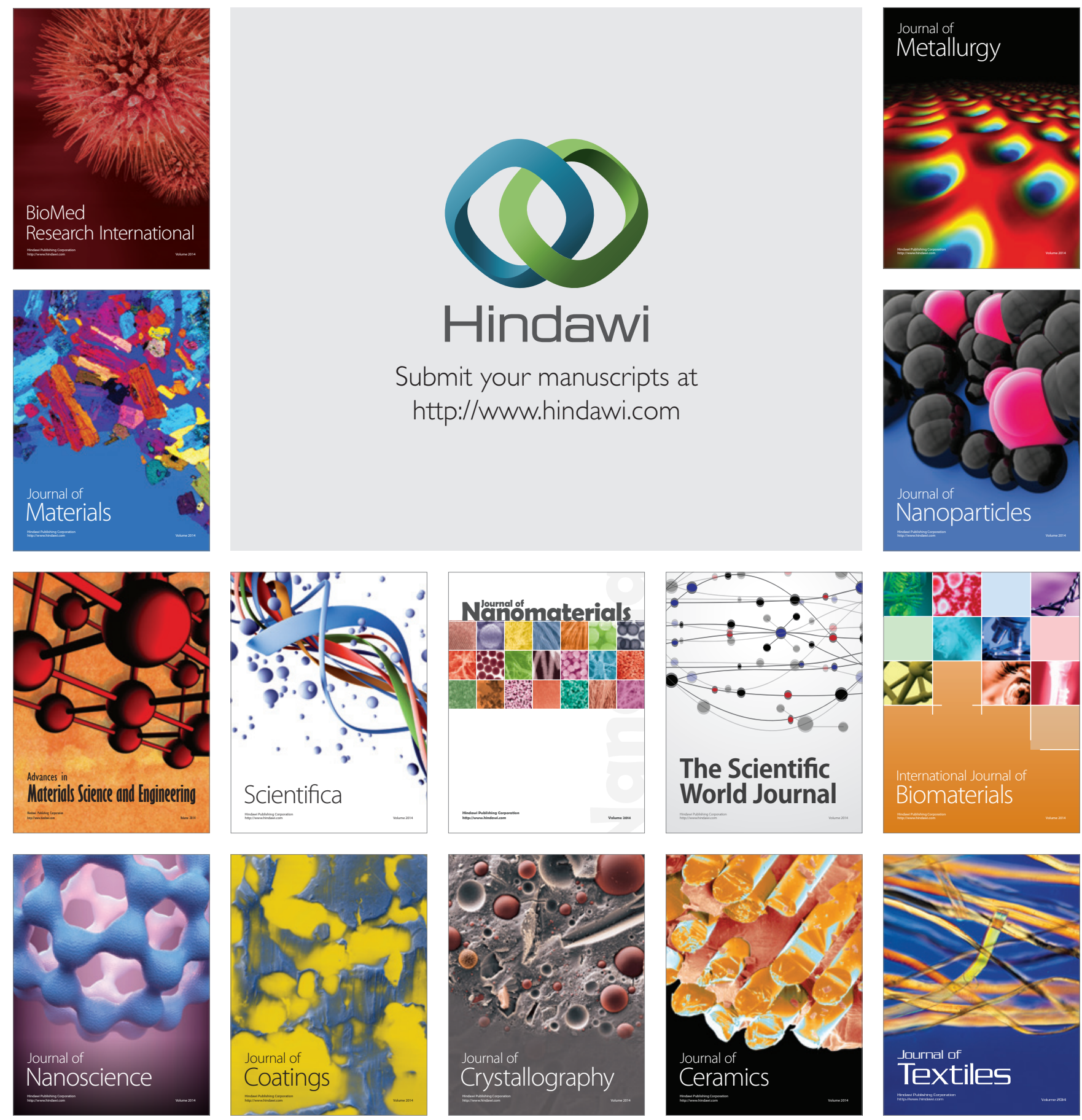